\section{Optical Absorption Spectra of Vanadyl Ion (Impurities) in Three Host Crystal Compounds}

O. P. Agarwal

Physics Section, Engineering College, Aligarh Muslim University, Aligarh-202001, India

\section{Prem Chand}

Department of Physics, Indian Institute of Technology, Kanpur-208016, India

Z. Naturforsch. 40 a, 1164-1166 (1985); received August 19, 1985

Results of the optical absorption study of vanadyl ion doped in magnesium ammonium sulphate hexahydrate, rubidium sulphate and potassium dihydrogen phosphate single crystals at RT are reported. The nature of optical bands suggests a $\mathrm{C}_{4}$ r symmetry of the Vanadyl complexes in conformity with the EPR results. Powder EPR data and optical data are correlated to obtain the MO coefficients.

Vanadyl ions $\left(\mathrm{VO}^{2+}\right)$ occur coordinated to other groups both in solids and liquids, bringing the total coordination to five or six. Usually the metal ion is surrounded by a distorted octahedron of oxygen ligands. However, the behaviour of the unpaired electron in the vanadyl complexes is dominated by the strong $\mathrm{V}=\mathrm{O}$ bonding; as a result, most of the complexes possess very nearly a square pyramid geometry $\left(\mathrm{C}_{4 v}\right)$. The EPR and optical data can be correlated to establish the symmetry and the bonding of the vanadyl complexes. The EPR studies of $\mathrm{VO}^{2+}$ ion in Tutton's salts [1-5] have shown that in all these hosts, substitutional pentahydrate vanadyl complexes $\left[\mathrm{V}=\mathrm{O}\left(\mathrm{H}_{2} \mathrm{O}\right)_{5}\right]^{2+}$ are formed. These complexes have been found to show very nearly $\mathrm{C}_{4 v}$. symmetry on the basis of optical absorption studies [6-8].

The results of single crystal EPR studies of $\mathrm{VO}^{2+}$ ions doped in Magnesium Ammonium Sulphate Hexahydrate [MASH] $\left(\mathrm{Mg}\left(\mathrm{NH}_{4}\right)_{2}\left(\mathrm{SO}_{4}\right)_{2} \cdot 6 \mathrm{H}_{2} \mathrm{O}\right)$ [1], Rubidium Sulphate [RS] $\left(\mathrm{Rb}_{2} \mathrm{SO}_{4}\right)$ [9] and Potassium dihydrogen phosphate [KDP] $\left(\mathrm{KH}_{2} \mathrm{PO}_{4}\right)[10]$ have shown the presence of multiple sites of the substitutional complexes in these hosts. In RS and KDP the substitutional vanadyl complexes are associated with the appropriate charge compensating vacancies. Since usually the single crystal EPR spectrum of the vanadyl ion consists of lines from multiple sites, making the spectrum much more complex, the spin Hamiltonian parameters (SHP) are more easily determined by crushing the crystal into a fine powder and by recording the polycrystalline spectrum $[6-9,11]$. The powder EPR spectra recorded by us on a Varian E- 109 X-band EPR spectrometer both at RT and at LNT have shown that in MASH,

Reprint requests to Dr. Prem Chand, Department of Physics, Indian Institute of Technology, Kanpur-208016, India.
RS and KDP all the single crystal sites give identical spectra and possess an axial symmetry (i.e. $g_{x}=g_{y}$ and $A_{x}=A_{y}$ ) within the experimental limits. The SHP do not vary appreciably down to LNT. Our powder EPR data (Table 1) are in good agreement with the single crystal EPR data (using $g_{\|}=g_{z}, g_{\perp}=\left(g_{x}+g_{y}\right) / 2, A=A_{z}$ and $\left.A_{\perp}=\left(A_{x}+A_{y}\right) / 2\right)[1,9,10]$.

The optical absorption spectra of $\mathrm{VO}^{2+}$ doped single crystals of MASH, RS and KDP, recorded on a Beckman DU-6 spectrophotometer at RT, have shown three absorption bands at: 13000, 16100, > 30000; 13200, 16200, $\sim 30000$ and 12000, 15300, 30000 (expressed in $\mathrm{cm}^{-1}$ ) respectively (Figure 1). The first one being relatively intense, the second appears as a shoulder to it; the third is a very intense and broad band (Figure 1). Similar bands have earlier been observed in $\mathrm{MgNH}_{4} \mathrm{PO}_{4}, \quad 6 \mathrm{H}_{2} \mathrm{O}$ (struvite) [11] and in some Tutton's salts [6-8]. From the relative positions and the nature of the absorption bands [12] the first two bands could be attributed to characteristic $d-d$ bands of the vanadyl complexes with a tetragonal symmetry, and the third intense band towards shorter wavelengths to the characteristic charge transfer band [12]. In the present case the first two bands have been assigned to the following expected $\mathrm{d}-\mathrm{d}$ transitions, in order of increasing energy; ${ }^{2} \mathrm{~B}_{2 g} \rightarrow{ }^{2} \mathrm{E}_{g}\left(\Delta_{\perp}\right)$ and ${ }^{2} \mathrm{~B}_{2 g} \rightarrow{ }^{2} \mathrm{~B}_{1 g}\left(\Delta_{\|}\right)$.

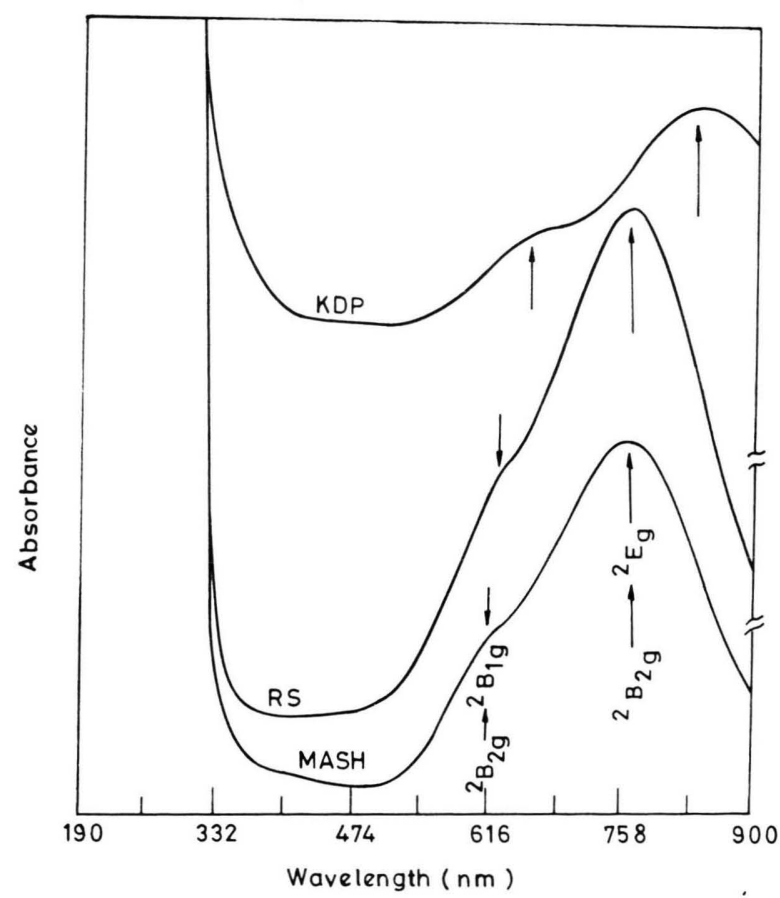

Fig. 1. The optical absorption spectra of $\mathrm{VO}^{2+}$, doped in MASH, RS and KDP single crystals at RT. The position of the absorption peaks and their assignments are shown.

0340-4811/85/1100-1164\$01.30/0. - Please order a reprint rather than making your own copy. 
Table 1. Powder EPR data and MO parameters of $\mathrm{VO}^{2+}$ in MASH, RS and KDP.

\begin{tabular}{lllllll}
\hline Host & Temperature & $g_{\|}$ & $g_{\perp}$ & $A_{\|}\left(10^{-4} \mathrm{~cm}^{-1}\right)$ & $A_{\perp}\left(10^{-4} \mathrm{~cm}^{-1}\right)$ \\
\hline \multirow{2}{*}{ MASH } & RT & 1.937 & 1.988 & 190 & 74 \\
RS & LNT & 1.937 & 1.988 & 193 & 75 \\
& RT & 1.931 & 1.978 & 178 & 68 \\
KDP & LNT & 1.933 & 1.980 & 180 & 70 \\
& RT & 1.923 & 1.976 & 204 & \multicolumn{2}{c}{77} \\
& LNT & 1.924 & 1.977 & 206 & & 78 \\
& \multicolumn{2}{c}{$P\left(10^{-4} \mathrm{~cm}^{-1}\right)$} & $K$ & $\beta_{1}^{2}$ & $\gamma_{1}^{2}$ & $\left\langle r^{-3}\right\rangle(\mathrm{au})$ \\
\hline MASH & RT & -127 & 0.85 & 0.77 & 0.55 & 2.92 \\
RS & RT & -120 & 0.84 & 0.85 & 0.86 & 2.75 \\
KDP & RT & -137 & 0.83 & 0.88 & 0.93 & 3.15 \\
\hline
\end{tabular}

(Error in $g$ values is \pm 0.002 and $A$ values is \pm 2 ).

Under the electric dipole selection rules, valid for a tetragonal $\mathrm{C}_{4 r}$ symmetry, only transition $\Delta_{\perp}$ is allowed and is expected to be relatively strong while transition $\Delta$ and a third expected transition, ${ }^{2} \mathrm{~B}_{2 g} \rightarrow{ }^{2} \mathrm{~A}_{1 g}(\Delta)$, are normally forbidden and are allowed vibronically [12]. These latter bands are expected to be relatively weak, which indeed is the case. The third expected d-d band $(\Delta)$ is probably not detectable due to being masked by the intense charge transfer band. These observations support the assumption that the vanadyl complexes in the hosts under study possess very nearly a tetragonal $\left(\mathrm{C}_{4 i}\right)$ symmetry in conformity with EPR observation.

The optical absorption and powder EPR data have been correlated to obtain molecular orbital (MO) parameters for the vanadyl ion from the following relations [12]:

$$
\begin{aligned}
& g_{\|}=2\left(1-\frac{4 \lambda \beta_{1}^{2}}{\Delta_{\|}}\right), \quad g_{\perp}=2\left(1-\frac{\lambda \gamma_{1}^{2}}{\Delta_{\perp}}\right), \\
& A_{\|}=-P\left[\left(\frac{4}{7}+K\right)+\frac{8 \lambda \beta_{1}^{2}}{\Delta_{\|}}+\frac{6}{7}\left(\frac{\lambda \gamma_{1}^{2}}{\Delta_{\perp}}\right)\right], \\
& A_{\perp}=P\left[\left(\frac{2}{7}-K\right)-\frac{11}{7}\left(\frac{\lambda \gamma_{1}^{2}}{\Delta_{\perp}}\right)\right],
\end{aligned}
$$

where $\beta_{1}^{2}$ and $\gamma_{1}^{2}$ are the bonding coefficients, $\lambda$ is the spin orbit coupling constant for the vanadyl ion, $\Delta_{\perp}$ and $\Delta_{\|}$are the energy splittings as defined earlier,

$$
P\left(=2.0023 g_{\mathrm{N}} \beta \beta_{\mathrm{N}}\left\langle r^{-3}\right\rangle\right)
$$

is the dipolar coupling constant and $K$ is the Fermi contact parameter. A reasonable value of $\lambda=170 \mathrm{~cm}^{-1}$ is usually taken for the vanadyl ion [13]. By making use of the powder EPR data (Table 1) and observed optical absorption band positions, the equations (1) have been solved to get $P, K, \beta_{1}^{2}, \gamma_{1}^{2}$ and $\left\langle r^{-3}\right\rangle$ as summarized in Table 1.

The calculated values for $\left\langle r^{-3}\right\rangle$ are rather low compared to the theoretical free ion value $\left\langle r^{-3}\right\rangle=3.67$ [14]. Such a reduction in $\langle r\rangle^{-3}$ in the solid state is often attributed to bonding effects. The parameters $\beta_{1}^{2}$ and $\gamma_{1}^{2}$ indicate the inplane " $\sigma$ " and out-of plane " $\pi$ " bonding, respectively, and parameters $\left(1-\beta_{1}^{2}\right)$ and $\left(1-\gamma_{1}^{2}\right)$ indicate the covalent nature. The values of these parameters indicate that both the inplane " $\sigma$ " and the out-of plane " $\pi$ " bondings are of partly covalent nature, the latter being more covalent in the vanadyl complexes under study. It can be seen from Table 1 that the covalency increases from KDP to MASH. Since in MASH the ligands to $\mathrm{VO}^{2+}$ are water molecules and in $\mathrm{RS}$ and $\mathrm{KDP}$ the ligands are oxygens from $\mathrm{SO}_{4}$ and $\mathrm{PO}_{4}$ groups, respectively, it appears that the water ligands are less electronegative than the $\mathrm{SO}_{4}$ and $\mathrm{PO}_{4}$ ligands, and that the $\mathrm{PO}_{4}$ ligands are the most electronegative ones. The parameter $K$ is related to the unpaired electron density at the vanadium nucleus and typically varies from 0.60 to 0.85 for various vanadyl complexes. This parameter shows extreme sensitivity to the deformations of the electron orbitals of the central vanadium ion. The values of the MO coefficients obtained for the $\mathrm{VO}^{2+}$ complexes in MASH, RS and KDP are close to those obtained for various axial vanadyl complexes in a variety of crystalline hosts $[6-8 ; 11-13]$. It may therefore be concluded that vanadyl complexes in MASH, RS and KDP possess a "very nearly" $\mathrm{C}_{4 r}$ symmetry and that the EPR and optical results are in conformity with each other. 
[1] M. Narayana, S. G. Sathyanarayan, and G. S. Sastry, Mol. Phys. 31, 203 (1976).

[2] R. H. Borcherts and C. Kikuchi, J. Chem. Phys. 40, 2270 (1964).

[3] R. S. Saraswat and G. C. Upreti, J. Phys. Soc. Japan 44, 1142 (1978); R. S. Saraswat and G. C. Upreti, Phys. Stat. Sol. (b) 122, K 169 (1984).

[4] S. Kasthurirengan and S. Sundarajan, J. Magn. Reson. 19, 357 (1975).

[5] V. K. Jain and T. M. Srinivasan, Ind. J. Pure Appl. Phys. 16, 796 (1978); Z. Naturforsch. 329, 1068 (1977).

[6] S. V. J. Lakshman and T. V. Krishna Rao, Sol. State Commun. 49, 567 (1984).

[7] J. L. Rao and K. Purandhar, Sol. State Commun. 33, 363 (1980).
[8] V. Somaseharam and Y. P. Reddy, Sol. State Commun. 53,695 (1985)

[9] V. K. Jain, V. P. Seth, and R. K. Malhotra, J. Phys. Chem. Sol. 45, 529 (1984).

[10] R. P. Kohin, J. Zakelj, and V. K. Jain, J. Chem. Phys. 68, 778 (1978).

[11] O. P. Agarwal and Prem Chand, Sol. State Commun. 52, 417 (1984).

[12] C. J. Ballhausen and H. B. Gray, Inorganic Chem. 1, 111 (1962).

[13] D. Kivelson and S. K. Lee, J. Chem. Phys. 41, 1896 (1964).

[14] R. M. Golding, Applied Wave Mechanics, D-VanNostrand Co. Ltd., London 1969, p. 303. 